\title{
Introduction to Biomaterials
}

\author{
Donglu Shi
}

\section{Editor}




\section{Published by}

Tsinghua University Press

Xueyan Building

Tsinghua University

Beijing, 100084, P.R. China

World Scientific Publishing Co. Pte. Ltd.

5 Toh Tuck Link, Singapore 596224

USA office: 27 Warren Street, Suite 401-402, Hackensack, NJ 07601

UK office: 57 Shelton Street, Covent Garden, London WC2H 9HE

\section{Library of Chinese Version Cataloging-in-Publications Data(2005) No. 031443 Introduction to Biomaterials $\quad$ D. L. Shi \\ ISBN: 7-302-10807-2 \\ I. Bio... II. Shi... III Biomaterials-Colleges and Universities-Textbook-in Eng1ish IV. Q81}

\section{British Library Cataloguing-in-Publication Data}

A catalogue record for this book is available from the British Library.

\section{INTRODUCTION TO BIOMATERIALS}

Copyright $(0) 2006$ by Tsinghua University Press and World Scientific Publishing Co. Pte. Ltd.

All rights reserved. This book, or parts thereof, may not be reproduced in any form or by any means, electronic or mechanical, including photocopying, recording or any information storage and retrieval system now known or to be invented, without written permission from the Publisher.

For photocopying of material in this volume, please pay a copying fee through the Copyright Clearance Center, Inc., 222 Rosewood Drive, Danvers, MA 01923, USA. In this case permission to photocopy is not required from the publisher.

ISBN $\quad 7-302-10807-2 / Q \cdot 47$

ISBN $\quad 981-256-627-9$

Printed in China. 


\section{Introduction}

In recent years, there has been increasing emphasis on materials applications in biomedical areas. However, the term "biomaterials" may have encountered different interpretations both in materials science and clinical medicine. Here we define a biomaterial as a synthetic material used to replace part of a living system or to function in intimate contact with living tissue. A biomaterial is different from a biological material, such as bone, that is produced by a biological system. Biomaterials can be categorized into different types in terms of their structural, chemical, and biological characteristics, for example, as in ceramics, glasses, and polymers with a varied degree of bioactivity. Bioma- terials represent an interdisciplinary research area that requires sufficient know- ledge of three different fields: (1) materials science and engineering processing-structure-property interrelationship of synthetic and biological materials including metals, ceramics, polymers, composites, tissues; (2) biology and physiology cell and molecular biology, anatomy, animal and human phy- siology, and (3) clinical sciences dentistry, ophthalmology, orthopedics, plastic and reconstructive surgery, cardiovascular surgery, neurosurgery, immunology, histopathology, experimental surgery, veterinary medicine and surgery.

At present, bioactive materials include some calcium phosphate compounds, bioactive glasses, bioactive glass-ceramics, bioactive ceramic coatings deposited on metal substrates, composites containing bioactive ceramic phase $(\mathrm{s})$, etc. A characteristic feature common to these materials is that they bond to human bone with no fibrous tissue at the interface. Commonly, bioactivity has been defined as an ability to form an adherent interface with the tissues. The interface possesses considerable mechanical bonding and its cohesive strength is equivalent to that of the tissue and implant material. The reactions at the interface are complicated by the different responses of various types of materials.

Calcium-phosphate-based bioceramics have been in use in medicine and 
dentistry for more than 20 years. The interest in one group member, hydroxyapatite (HA), arises from its similarity to bone apatite, the major component of the inorganic phase of bone, which plays a key role in the calcification and resorption processes of bone. The stable phases of calcium phosphate ceramics depend significantly on temperature and the presence of water, either during materials processing or in the application environment. At body temperature, only two calcium phosphates are stable when in contact with aqueous media such as body fluid ( BE). The term apatite describes a family of compounds having similar structures but not necessarily having identical compositions. Biological apatites constitute the mineral phase of calcified tissues such as bone, dentin and enamel in the body and also some pathological calcifications. Biological apatites, which comprise the mineral phases of calcified tissues (enamel, dentin, bone) and of some pathological calcifications (e.g. human dental calculi, salivary stones), are usually referred to as calcium hydroxyapatite. The biological apatites are similar to synthetic hydroxyapatite (HA) but they differ from HA in composition, stoichiometry, and physical and mechanical properties. Biological apatites are usually calcium-deficient as a result of various substitutions in regular $\mathrm{HA}$ lattice points. $\mathrm{HA}$, as noted before, is a compound of a definite composition, $\mathrm{Ca}_{10}\left(\mathrm{PO}_{4}\right)_{6}(\mathrm{OH})_{2}$, and a definite crystal structure. HA has been routinely used in orthopaedic surgery in both powder and bulk forms.

The current trend of developing bioactive materials has motivated an increasing need for developing biomaterials in a variety of applications. Indeed, it is clear that to achieve highly bioactive and mechanically compatible artificial materials for hard tissue prosthetics and tissue engineering(TE), it is necessary to seek for novel synthesis routes by which ideal materials can be developed with required bioactivity, porosity, microstructure, and mechanical properties. In the past, great efforts have been focused on developing metallic, ceramic, and polymer materials that could interfacially and bioactively bond to hard tissues. For instance, coating of bioactive materials onto various dense alloys and ceramics has been an effective approach in the development of bio-composite materials. However, as a result of extremely high strength and rigidity of these materials, they are not mechanically compatible with the hard tissues. On the other hand, the commonly used bioactive material HA is extremely weak in its porous form and cannot be used as structural bones. In these conventional materials, the properties cannot be easily, structurally and synthetically adjusted for bone implant. It is, therefore, highly desirable to develop a variety of bioactive materials whose structures and properties can be simulated to a real human bone.

Bioactive HA film has been coated onto porous ceramic substrates such as 
an industrial porous alumina (or reticulated alumina). Alumina is not only bio-inert but also mechanically strong which makes it an ideal substrate for bone substitute. The high porosity is achieved via a sponge technique by which both pore size and density can be changed easily. The bioactivity is induced by coating a HA film onto the inner pore surfaces of the reticulated alumina. Although the ceramic coating has been established on some dense substrates, it poses a great challenge in coating inner surfaces of pores as the coating area is not only partially enclosed in the material's matrix but also curved. No previous studies have so far been reported on coating inner surfaces of small-diameter pores ranging from $0.1-1.0 \mathrm{~mm}$. The key materials processing issues dealt with in this book include precursor chemistry, coating procedures, synthesis of coated component, interface structure study, film adhesion strength testing, and mechanical properties of the component. In addition, to determine the applicability of the coated component in hard tissue prosthetics, a bioactivity study has been presented. By immersing the synthetic HA into simulated body fluid ( SBF), the bioresponse has been measured for a variety of samples with different processing conditions. Significant conclusive remarks have been drawn from these extensive experiments that this novel approach has shown great promise in synthesizing structural artificial bones for hard tissue prosthe-tics. The fabricated components can be designed and synthesized to have similar properties comparable to human bones in terms of porosity, mechanical strength, and bioactivity. Furthermore, fundamental aspects of this research are centered on the effects of structural characteristics of HA on bioactivity. Based on extensive infrared spectroscopy (IR) and X-ray diffraction (XRD) experimental data, it has been found that the bioactivity of HA is sensitively controlled by the structural crystallinity of the HA and its specific surface area. A physical model has been developed to interpret the observed phenomena.

Polymers have been widely used as biomaterials for the fabrication of medical device and tissue engineering scaffolds. Naturally occurring polymers, synthetic non-biodegradable and synthetic biodegradable polymers are the main types of polymers used as biomaterials. Naturally occurring polymers, such as collagen, chitin and starch, are an important class of biomaterials due to their biodegradation characteristics and plentiful availability. Synthetic polymers represent the majority of the polymer biomaterials - from traditional engineering plastics to newly engineered biomaterials for specific biomedical applications. Although most synthetic non-biodegradable polymers were originally designed for non-biomedical use, they are widely used as biomaterials mainly because of their desirable physical-mechanical properties. There are still no newly engineered biomaterials, which can replace these "old" polymers. A good example is Polymethyl metacrylate (PMMA) bone cement, which has been used since 
1943 and is still being widely used clinically nowadays. Synthetic biodegradable polymers have attracted much attention in the last decade because the biodegradable polymers can be eliminated from the human body after fulfilling their intended purpose and therefore a second surgery can be avoided. Also, the emergence of tissue engineering has demanded the use of biodegradable scaffolds for the regeneration of tissues and organs. A lot of biodegradable polymers are derived from the traditional polymers by introducing non-stable chemical linkages, such as hydrolysable ester bonds.

The use of biomaterials in orthopedic surgeries represents a significant challenge to biomaterials scientists. Biomaterials for orthopedic use will have to possess sufficient mechanical properties for sharing or bearing the load. They will also need to be able to bond to host bone (bone-bonding). Ideally, the biomaterials will be gradually replaced by newly formed bone during the wound healing process. One of the solutions is to use bioactive ceramics, such as calcium phosphate and Bioglass ${ }^{\mathrm{TM}}$, to reinforce polymers so that the mechanical properties and the bone-bonding properties of the polymer can be improved.

This book gives a comprehensive introduction to most of the important biomaterials including ceramics, metals, and polymers from a fundamental point of view, which is suitable for both undergraduate and graduate students. Part I mainly deals with critical issues in bioceramics and metals in the areas of synthesis methods, characterization techniques, mechanical property analysis, heat treatment, and in vitro bioactivity test. Most recent experimental results on the coating of HA onto porous ceramics and their bioactivity study are included in Chapter 2 through Chapter 7 . These chapters should interest many researchers in this field. An introduction to metallic biomaterials is included in Chapter 8. Part II addresses the important problems in polymers and their applications in medical fields. Part II is entirely dedicated to tissue engineering, which is closely related to Parts I and II. Not only are these chapters well referenced with the most up-to-date publications, but also followed with exercise problems and questions for classroom lecturing.

Biomaterials research has been advancing rapidly in recent years, particularly in the field of tissue engineering. The research addresses the critical issues in medical applications including bioactivity, compatibility, toxicity, and mechanical properties of all types of biomaterials. In the biomedical applications, traditional materials science and engineering have to deal with new challenges in the areas of synthesis, microstructure development, and biological, chemical, and physical behaviors, since medical needs place new demands in these respects.

The most fascinating development in biomaterials is to be found in tissue engineering, and involves the direct use of material within a biological system. 
Today, tissue-engineered skin is already available on the market; and tissue-engineered cartilage is undergoing clinical trials and should be available within a few years. Temporary liver-assistance devices are also being clinically tested. Tissue-engineered bone is not very far behind. Up to now, investigators have attempted to build bone, liver, arteries, bladder, pancreas, nerves, cartilage, heart valves, cornea, and various soft tissues. Despite significant progress in this field, a number of issues have arisen that force the commercial process to take a breather. By simply producing highly porous scaffold and seeding it with appropriate cell types, one cannot in most cases obtain the desired features of a normal tissue. The challenge for future tissue engineering concerns how subcellular structures dictate cell function. We need to know how to fabricate organ-scale structures with cellular resolution, integrating functional cells to yield a 3-D architecture for tissue function. This is necessary to integrate the tissue into patients with the desired vascularization and controlled immune responses.

A typical tissue engineering device comprises cells and scaffolds. Typically, in the construction, biological materials (cells and cellular products) provide the biological function whereas synthetic materials give the structural support. Ideally, the interaction results in the integration of the device with the host, maintenance of the biological function, and control of signals between device and host. Biomaterials have been used as supporting scaffold for the growth of cellular components. The materials can vary from polymers to bioceramics. One interesting trend in developing biomaterials for scaffolds is to design specialized biomaterials. For example, one can design a material that erodes to naturally occurring byproducts in vivo (temporary scaffold), incorporate bioactive moieties to facilitate tissue in-growth and host response, block undesirable biological phenomena, and alter material properties in response to environmental stimuli ( smart materials). The scaffold should provide the physicochemical signals to control cellular interactions, give structural support, and also provide sites for cell attachment, migration, and tissue in-growth. Biomaterial microstructure is often dictated by process parameters such as the choice of solvent in phase separation, doping with leachants, controlled ice crystal formation and subsequent freeze-drying to create pores. Almost all these techniques are very sensitive to processing parameters. The other important issue in biomaterials is the scaffold chemistry patterning. Scaffold may incorporate specific bioactive chemical moieties to direct cell adhesion, migration, and tissue ingrowth and repair. A precisely defined scaffold depends on the development of a novel biomaterial tailored to specific physicochemical material properties and structures.

Despite extensive research activities in biomaterials and tissue engineering, there have been a few comprehensive textbooks that systematically introduce 
the fundamental concepts in all types of materials including metallic, ceramic, and polymer materials in terms of their synthesis, structure, and bioactive behaviors. Further, closely related materials applications in tissue engineering are not entirely combined with the introduction of specific biomaterials in a textbook.

University students and researchers can benefit from this book in diversified backgrounds such as orthopedics, biochemistry, biomedical engineering, materials science, tissue engineering, and other related medical fields. Both undergraduate and graduate students will find the book a valuable reference not only on biomaterial, but also on tissue engineering-related topics, including biostructures and phase diagrams of complex systems, hard tissue prosthetics, novel biomaterials processing methods, and new materials-characterization techniques. Thus, it can serve as a comprehensive introduction to researchers in biomaterials science and engineering in general, and can also be used as a graduate-level text in related areas.

This book is written by several prominent scholars in the fields of biomaterials and TE. Chapters $1-7$ are mainly written by Dr. Donglu Shi on bioceramics based on his lecture notes from a biomaterials class that he has taught at the University of Cincinnati. Some of the recent experimental research results are obtained from his laboratory at the University of Cincinnati. His formal $\mathrm{PhD}$ student, Dr. Gengwei Jiang was intensely involved in these experiments and mainly responsible for the data presented in these chapters. Donglu Shi is most grateful to her dedicated work and appreciates the extensive previous results used in Chapters $1-7$. Dr. Xuejun Wen has not only written the chapters with Dr. Ning Zhang in Part III on tissue engineering, but also co-authored with Donglu Shi in writing Chapter 2 and Chapter 3 on the fundamentals of bioceramics. Dr. Yang Leng is a well known scholar in biomaterials and responsible for Chapter 8 on metallic materials. Finally, Dr. Qing Liu has authored Part II on bio-polymers. In Chapter 1 , part of the definitions of biomaterials is from a book chapter by Prof. M. Wang ("Biomaterials and Tissue Engineering" ed. D. Shi, Springer-TUP, 2004). We hope these chapters will provide timely and useful information for the progress of biomaterials and clinical applications. 


\section{Contents}

\section{Part I Bioactive Ceramics and Metals}

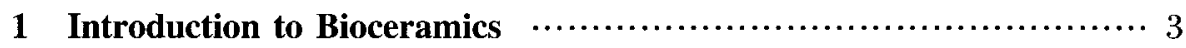

1.1 Bioactive Materials ….......................................... 3

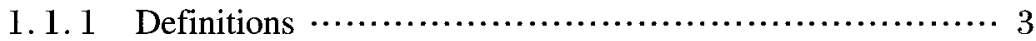

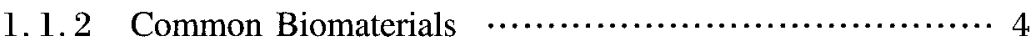

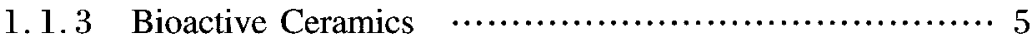

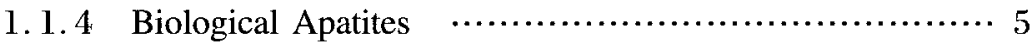

1. 1.5 Basic Requirements for Bone Implants …............. 6

1. 1.6 Coating of Hydroxyapatite on Porous Ceramics …...... 8

1.1.7 Biomaterials in Tissue Attachment …................... 11

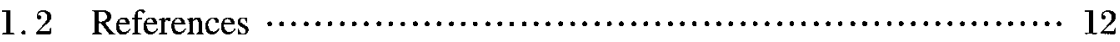

2 Bioactive Ceramics: Structure, Synthesis, and Mechanical

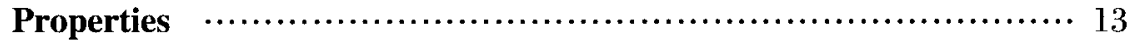

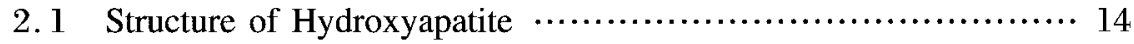

2.1.1 General Structure and Chemistry of Hydroxyapatite ….. 14

2.1.2 Structural Characteristics of Hydroxyapatite ….......... 15

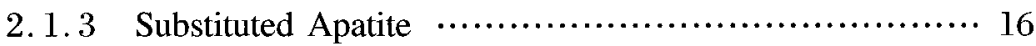

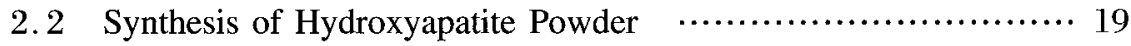

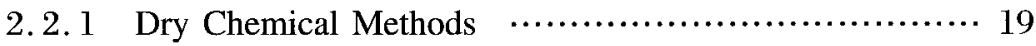

2.2.2 Wet Chemical Methods ….............................. 20

2.3 Mechanical Properties of Hydroxyapatite $\quad \cdots \ldots \ldots \ldots \ldots \ldots \ldots \ldots . . . \cdots 23$

2.4 Other Bioceramics ….......................................... 25

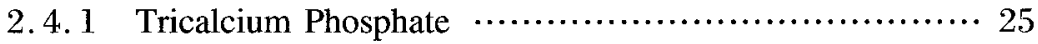

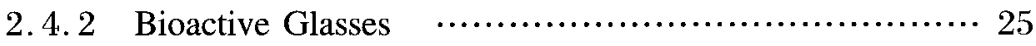

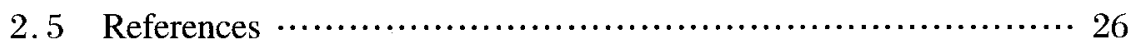

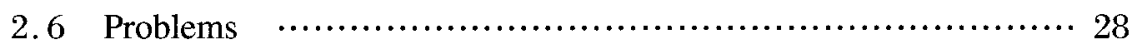

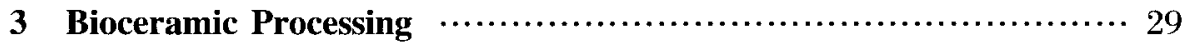

3. 1 Fabrication and Mechanical Properties of Porous Bioceramics $\cdots 30$ 


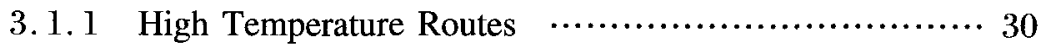

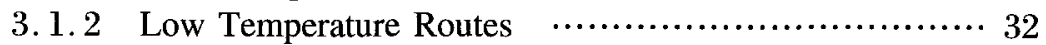

3.1.3 Rapid Prototyping Techniques

3. 1. 4 Mechanical Properties of Porous Bioceramics …....... 34

3.2 Coating of Bioceramic Thick Films on Bio-Inert Porous

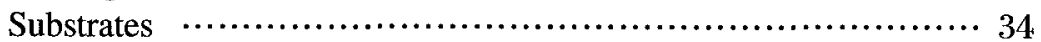

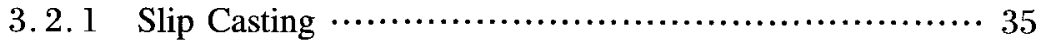

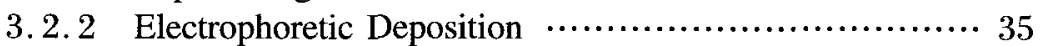

3.2.3 Bioceramic-Glass Slurry Method ….................. 36

3.2.4 Thermal Deposition ................................. 37

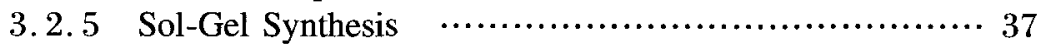

3.2.6 Biomimetic Growth ................................... 39

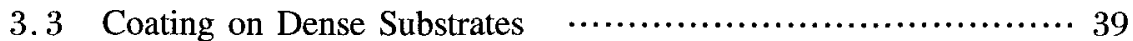

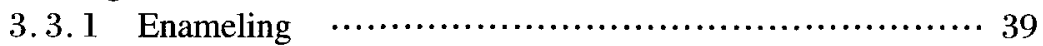

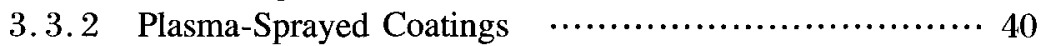

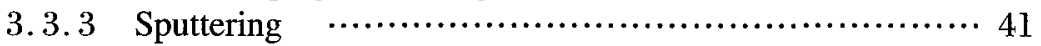

3. 4 Hydroxyapatite Coatings for Non-Hard Tissue Applications $\cdots \cdots 42$

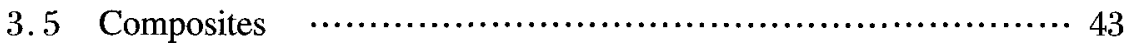

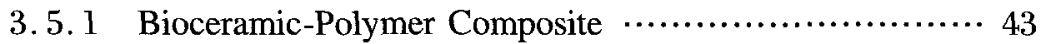

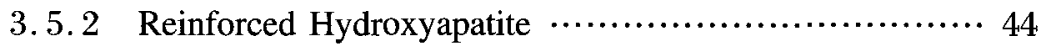

3.5.3 Hydroxyapatite and Tricalcium Phosphate Composite $\cdots 44$

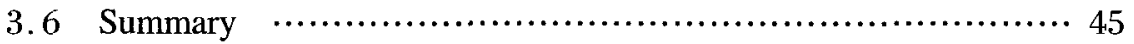

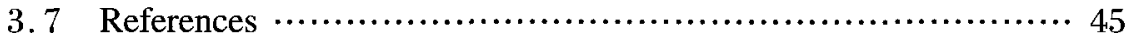

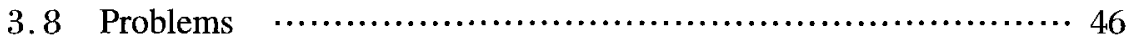

4 Coating of Hydroxyapatite onto Inner Pore Surfaces of the Reticulated

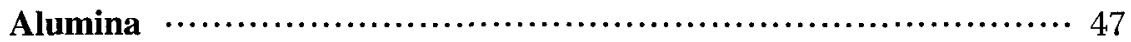

4. 1 Hydroxyapatite Coating Methods and Characterization $\ldots \ldots \ldots \ldots . .47$

4. 1. 1 Coating of Hydroxyapatite by the Hydroxyapatite-Glass

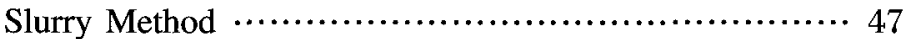

4. 1.2 Coating of Hydroxyapatite by a Thermal Deposition Method ............................................ 53

4. 1.3 Characterization of Hydroxyapatite Film …........... 53

4. 1.4 Coating of Hydroxyapatite Using Sol-Gel Synthesis ….. 57

4.2 Adhesion of Hydroxyapatite Film on Alumina Substrate ........ 58

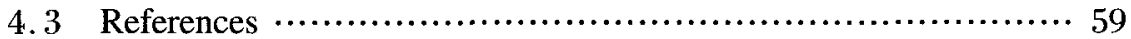

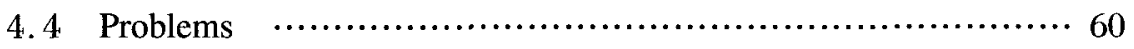

5 Properties and Characterization of Biomaterials $\ldots \ldots \ldots \ldots \ldots \ldots \ldots \ldots \ldots \ldots \ldots$

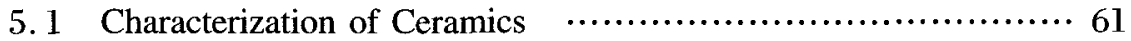




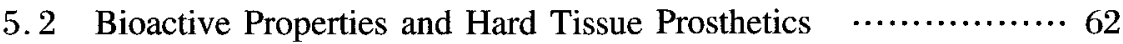

5.2.1 Bone Biology …..................................... 62

5.2.2 Critical Issues on Interfaces Between the Hard Tissue

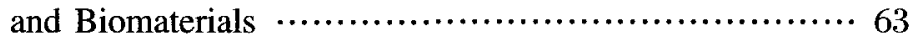

5.2.3 Factors that Influence Bioreactivity $\ldots \ldots \ldots \ldots \ldots \ldots \ldots .64$

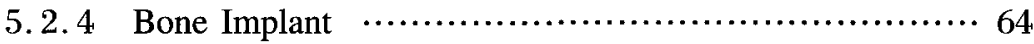

5.2.5 Bonding Mechanisms …............................. 65

5.2.6 In vitro Behavior of Hydroxyapatite …................. 66

5.3 Measurements of Growth and Dissolution of Hydroxyapatite

Ceramics ......................................................6 68

5.4 In vitro Test Conducted in This Reasearch …................... 68

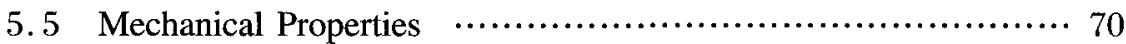

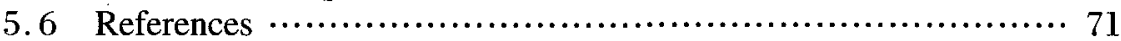

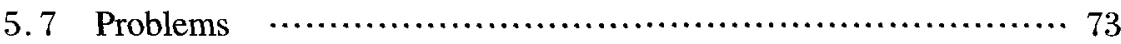

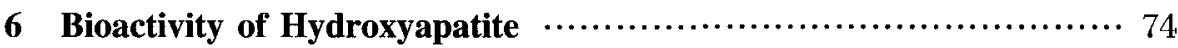

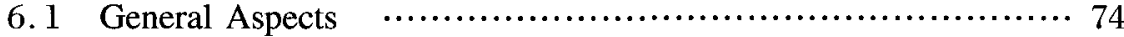

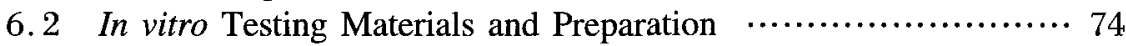

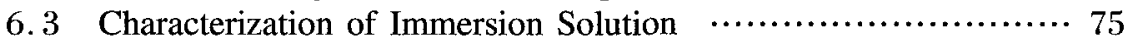

6.4 Morphology of the Reacted Surfaces $\ldots . \ldots \ldots \ldots \ldots \ldots \ldots \ldots \ldots \ldots \ldots . . \ldots 19$

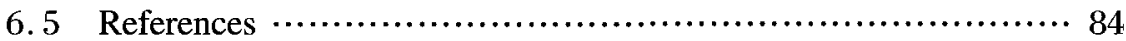

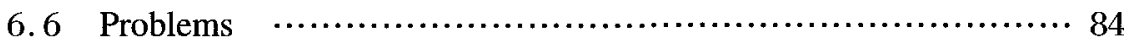

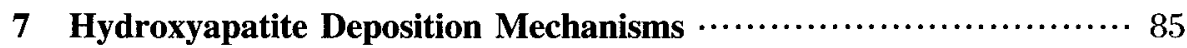

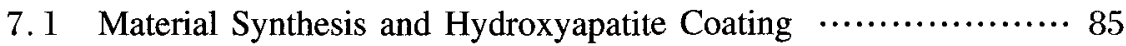

7.1.1 General Aspects on Chemistry, Structure, and

Thermal Behavior of Hydroxyapatite $\cdots \cdots \cdots \cdots \cdots \cdots \cdots \cdots \cdots$

7.1.2 Material Synthesis and Hydroxyapatite Coating $\cdots \cdots . . .86$

7.1.3 Coating of Hydroxyapatite onto Inner Surfaces

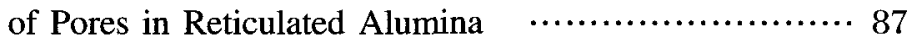

7. 1.4 Hydroxyapatite-Glass Slurry Method …................. 87

7. 1.5 Thermal Deposition and Sol-Gel Methods …............. 90

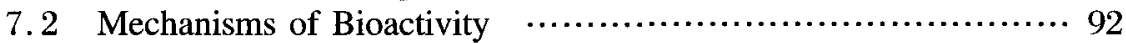

7.2.1 In vitro Biochemistry Behavior of Hydroxyapatite $\quad \ldots . .992$

7.2.2 Growth - Dissolution Mechanism …............... 94

7.2.3 Kinetics Models for Crystallization and Dissolution ..... 96

7.2.4 Experimental Determination of Reaction Mechanisms .. 99

7.2.5 Effect of Heat Treatment on Hydroxyapatite …........ 100

7.2.6 Structural Effect on Bioactivity ….................... 102

7.2.7 Temperature Effect on Bioactivity $\ldots \ldots \ldots \ldots \ldots \ldots \ldots \ldots \ldots . \ldots . \ldots 103$

7.2.8 Factors Contributing to Reactivity of Hydroxyapatite $\cdots 105$ 


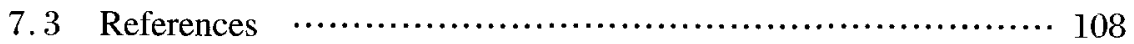

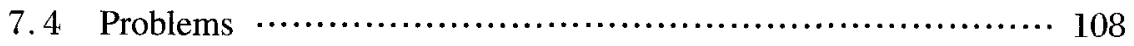

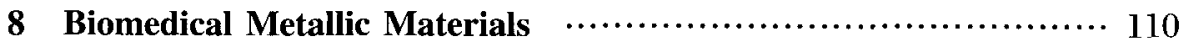

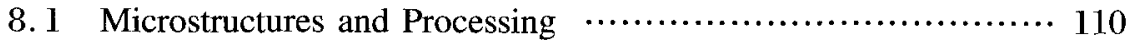

8.2 Corrosion Resistance of Metals ….......................... 115

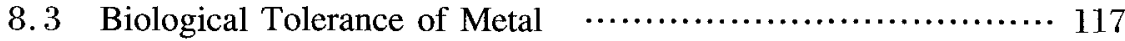

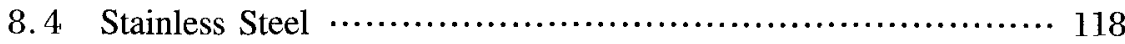

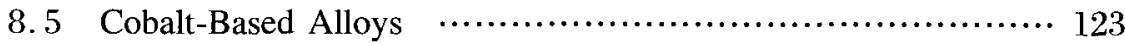

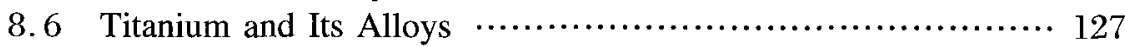

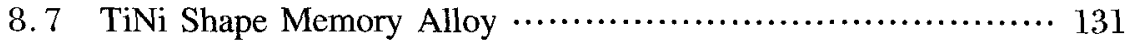

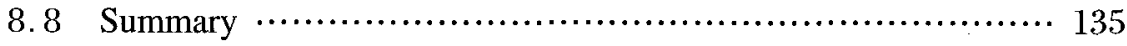

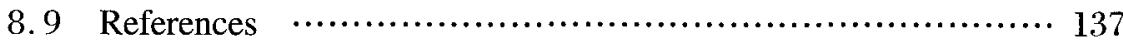

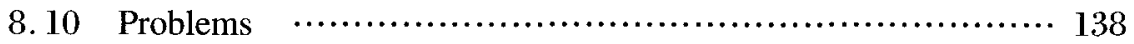

\section{Part II Polymeric Biomaterials}

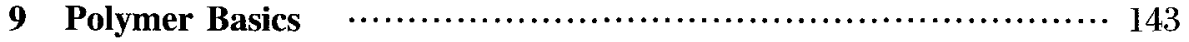

9. 1 Classification of Polymers ….............................. 143

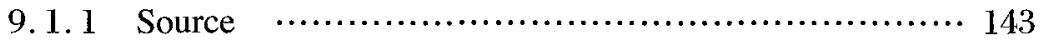

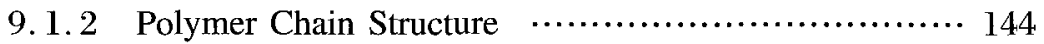

9.1.3 Polymer Thermal Behavior …...................... 146

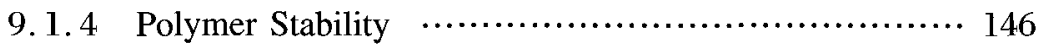

9.2 Characteristics of Polymer …................................ 147

9.2.1 Degree of Polymerization …....................... 147

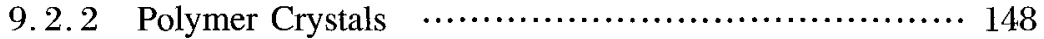

9.2.3 The Glass Transition Temperature and Melting

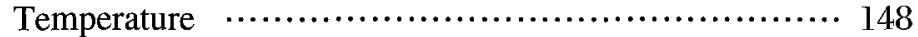

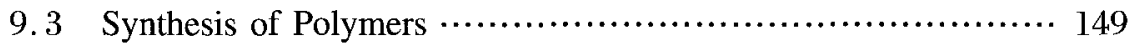

9.3. 1 Free Radical Polymerization .......................... 149

9.3.2 Condensation Polymerization

9.3.3 Other Types of Polymerization ….................... 154

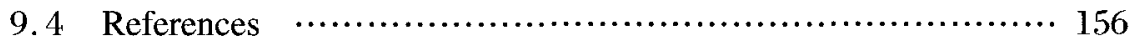

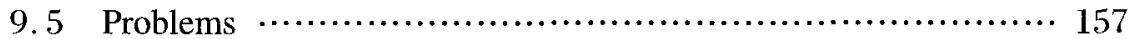

10 Naturally Occurring Polymer Biomaterials $\ldots \ldots \ldots \ldots \ldots \ldots \ldots \ldots \ldots \ldots \ldots \ldots \ldots \ldots$

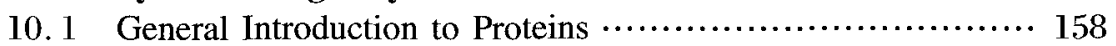

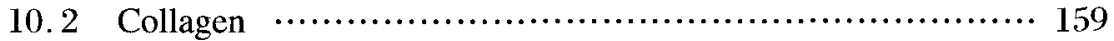

10.2. 1 Cross-Linking of Collagen ….................. 162

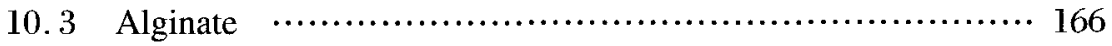




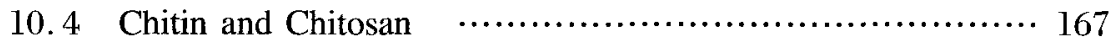

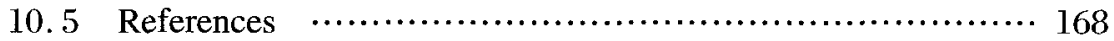

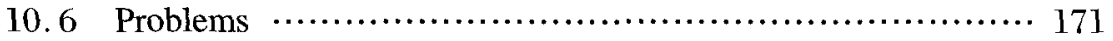

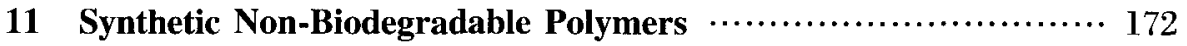

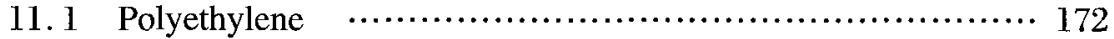

11.1.1 High Density Polyethylene ….................. 173

11. 1.2 Ultrahigh Molecular Weight Polyethylene …........ 174

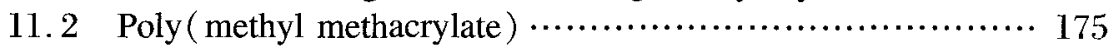

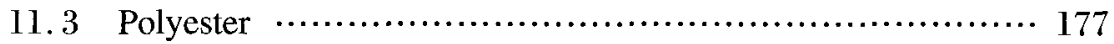

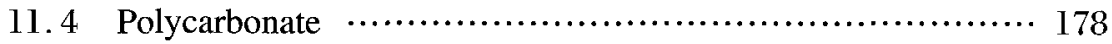

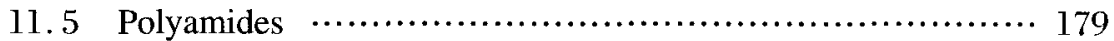

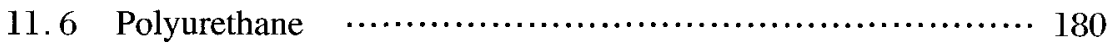

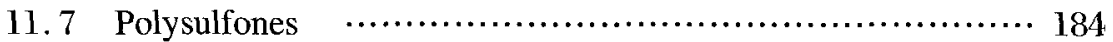

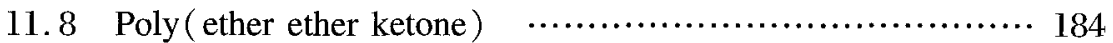

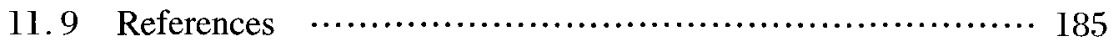

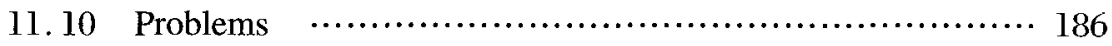

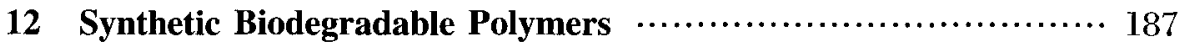

12.1 Aliphatic Polyester ….................................. 187

12.1.1 Poly (glycolide) ….............................. 189

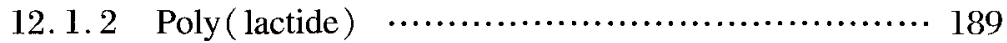

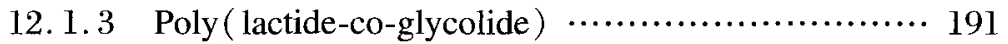

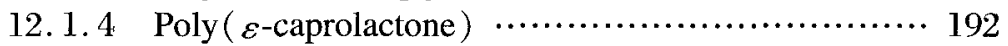

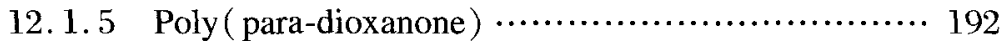

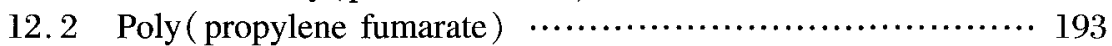

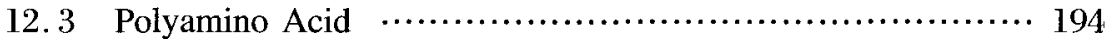

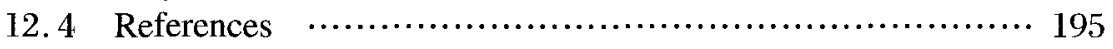

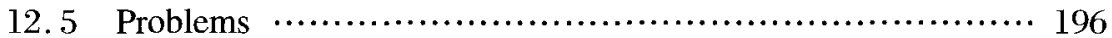

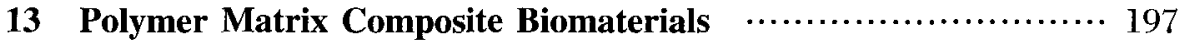

13. 1 Fiber Reinforced Composites ............................... 198

13.2 Filler Reinforced Composites .............................. 199

13.3 Methods to Improve the Interfacial Bonding Between

Phases in Composites ......................................... 200

13.3.1 Self-Reinforcement of Fiber/Polymer Composites $\cdots 200$

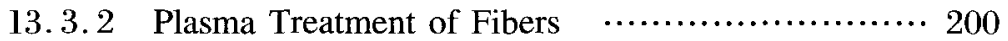

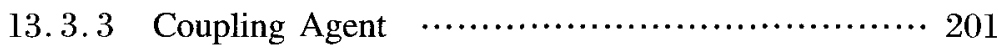

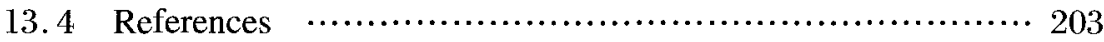

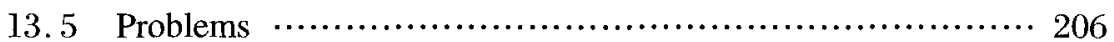




\section{Part III Tissue Engineering: A New Era of Regenerative Medicine}

14 Biomaterials for Tissue Engineering

14. 1 General Aspects of Biomaterials Used for Tissue Engineering

14.2 Representative Biomaterials Used for Tissue Engineering $\quad \cdots 212$

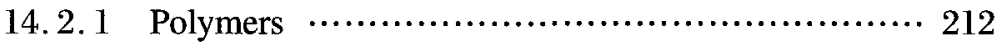

14.2.2 Bioceramics …................................ 213

14.3 Biomaterial Constructs for Tissue Engineering: Scaffolds $\cdots 214$ 14.3. 1 Definition and Requirements for Scaffolds Used in Tissue Engineering …............................ 215

14.3.2 Principles of Scaffold Design …................... 215

14.3.3 Scaffold Fabrication Technologies …............... 216

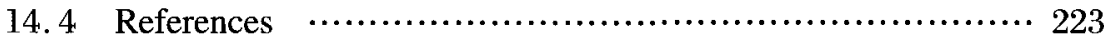

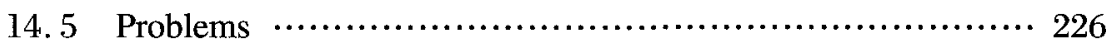

15 Cells and Biomolecules for Tissue Engineering $\cdots \cdots \cdots \cdots \cdots \cdots \cdots \cdots \cdots \cdots \cdots \cdots \cdots \cdots$

15.1 Cells for Tissue Engineering …........................... 227

15.2 Growth Factor Delivery in Tissue Engineering $\ldots \ldots \ldots \ldots \ldots \ldots . . .230$

15.3 Regulatory Matrix Proteins …............................ 232

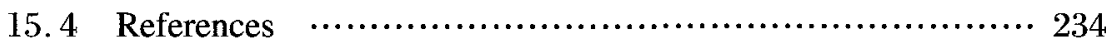

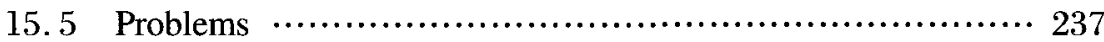

16 Transport and Vascularization in Tissue Engineering $\ldots \ldots \ldots \ldots \ldots 238$

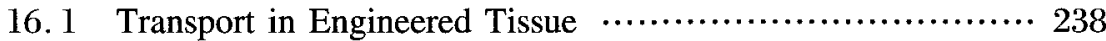

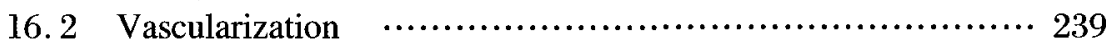

16.2. 1 New Blood Vessel Formation ...................... 239

16.2.2 Vascularization in Tissue Engineering $\cdots \ldots \ldots \ldots . . . .240$

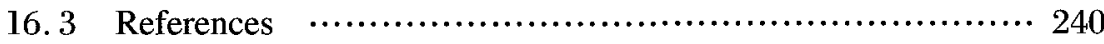

16. 4. Problems …................................................. 241

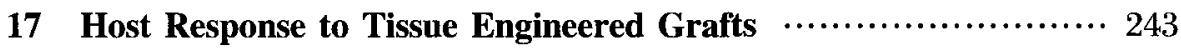

17. 1 The Foreign Body Response to Synthetic Components ….... 243

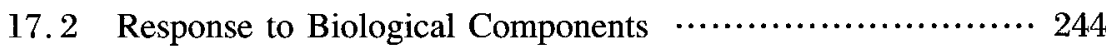

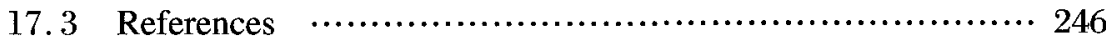

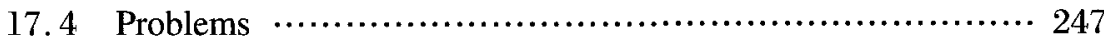


18 Other Important Issues and Future Challenges in Tissue

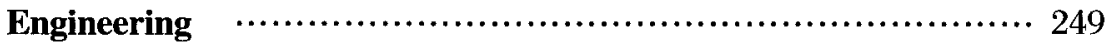

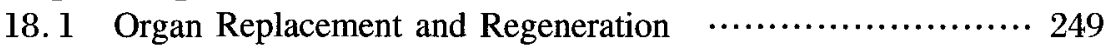

18.2 Organotypic and Histiotypic Models …...................... 250

18.3 Mechanotransduction …..................................... 251

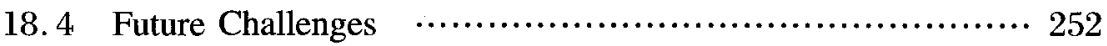

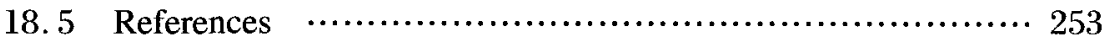

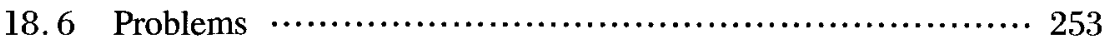

